\title{
Bioprosthetic Aortic Scallop Intentional Laceration to prevent latrogenic Coronary Artery obstruction (BASILICA): the first experience in Poland
}

\author{
Marcin Protasiewicz', Michał Kosowski', Grzegorz Onisk', Krzysztof Reczuch', Roman Przybylski², Piotr Kubler' \\ 'Department of Cardiology, University Hospital, Wrocław, Poland \\ ${ }^{2}$ Department of Cardiac Surgery, University Hospital, Wrocław, Poland
}

\author{
Correspondence to: \\ Grzegorz Onisk, MD, \\ Department of Cardiology, \\ University Hospital, \\ ul. Borowska 213 \\ 50-529 Wrocław, Poland, \\ phone: +48 503128344 , \\ e-mail: \\ gonisk46@gmail.com \\ Copyright by the Author(s), \\ 2021 \\ Kardiol Pol. 2021: \\ 79 (10): 1149-1150 \\ DOI: 10.33963/KP.a2021.0069 \\ Received: \\ March 22, 2021 \\ Revision accepted: \\ July 19, 2021 \\ Published online: \\ July 20, 2021
}

Transcatheter aortic valve implantation (TAVI) is a recognized treatment method for severe aortic stenosis as an alternative to surgical aortic valve replacement in high-risk patients [1]. However, despite being less invasive, TAVI may require specific considerations. Low coronary take-off, a small sinus of Valsalva diameter, and elongated aortic leaflets are associated with the risk of iatrogenic coronary obstruction during transcatheter heart valve (THV) deployment, whereby up to $50 \%$ of cases of such obstruction are fatal [2]. In these patients, the recently developed Bioprosthetic Aortic Scallop Intentional Laceration to prevent latrogenic Coronary Artery obstruction (BASILICA) technique may be considered.

The method involves bioprosthetic or native transcatheter leaflet laceration to prevent coronary obstruction during valve deployment. The lacerated leaflet creates a triangular space in front of the coronary ostium, which allows the coronary flow to be maintained after THV implantation. The procedure was reported to have a success rate of $95 \%$, with no cases of coronary obstruction [3].

We present a case of a 75-year-old woman with symptomatic severe aortic stenosis (aortic valve area, $0.7 \mathrm{~cm}^{2}$; average gradient, $63 \mathrm{~mm}$ $\mathrm{Hg}$ ), concomitant coronary artery disease (percutaneous coronary intervention of the left main artery in 2018), and low Euroscore II (1.47\%), in whom the Heart Team recommended TAVI due to frailty and advanced osteoarthritis.

Preoperative computed tomography revealed a low take-off of the left main artery $(8.2 \mathrm{~mm})$, left aortic leaflet $(10.8 \mathrm{~mm})$, the annulus diameter of $22.9 \mathrm{~mm}$, the sinus of Valsalva width of $26.2 \mathrm{~mm}$, and the virtual THV to coro- nary distance of $1.65 \mathrm{~mm}$. Considering a high risk of left main obstruction with a native leaflet, a decision to perform BASILICA was made.

TAVI was conducted under conscious sedation. The right ventricular pacing lead was placed through the right internal jugular vein. Judkins left (JL) 4.0 6F guide catheter was introduced through the right radial artery, and the left anterior descending artery was wired in case of emergency. Extra back-up (EBU) $3.56 \mathrm{~F}$ and IM 5F (mother-in-child) catheters were placed through the right common femoral artery. A multipurpose (MP) catheter introduced from the left common femoral artery was used to deliver a $20-\mathrm{mm}$ vascular snare, which was placed in the left ventricular outflow tract. A Piggyback ${ }^{\circledR}$ (Teleflex, Wayne, PA, USA) wire converter catheter with $300-\mathrm{cm}$ Astato $^{\circledR}$ (ASAHI, Irvine, SA, USA) wire inside, connected to an electrosurgical pencil, was placed via the IM catheter. The left coronary leaflet was punctured using 50-W energy, and the wire was snared and externalized contralaterally. Next, the wire was V-shaped and introduced until the $V$-bend reached the exact position of the leaflet puncture. The V-shaped wire was used to lacerate the leaflet using 70-W energy, with simultaneous pulling of the MP and EBU/IM catheters. After laceration, the Astato ${ }^{\circledR}$

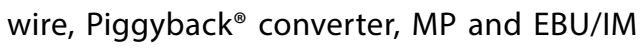
catheters were removed. The Medtronic Evolut Pro $^{\circledR}$ (Medtronic, Fridley, MN, USA) 26 valve was implanted via the left common femoral artery. Angiography with a repositioned JL catheter showed preserved blood flow to the left main artery.

The patient made an uneventful postoperative recovery and was discharged home after 7 days. She received single antiplatelet therapy 

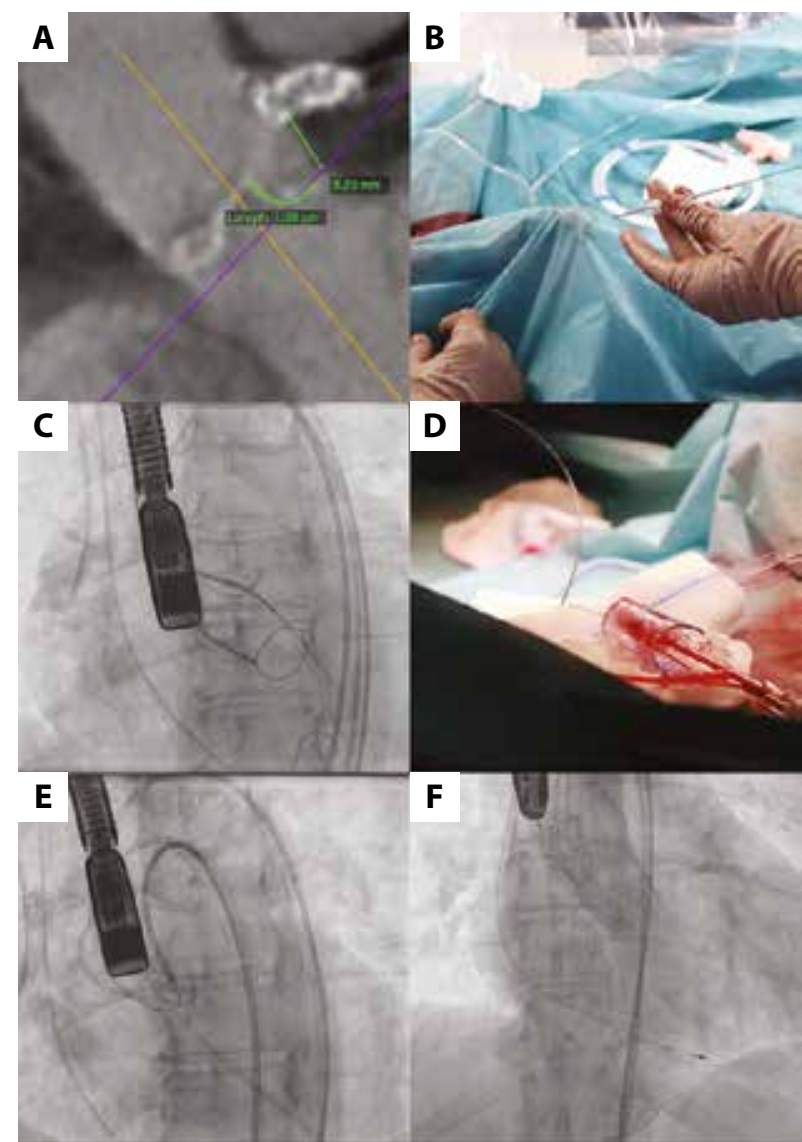

Figure 1. A. Computed tomography scan showing the left main artery take-off height of and leaflet length. B. A snare loop in left ventricular outflow trackt. C. Wire retrieval. D. V-shaped wire.

E. Snapshot of leaflet laceration sequence. F. Final outcome with a deployed valve and preserved coronary flow

according to the local protocol [4]. After 3 months, she was in New York Heart Association functional class I, and transthoracic echocardiogram showed good prosthetic valve function with mild aortic regurgitation.
We would like to underline that when performing BASILICA procedure one should be aware of possible complications, such as hemodynamic instability from leaflet laceration, nontarget Astato $^{\circledR}$ wire traversal (most commonly left atrial entry), and embolic debris release [5].

\section{Article information}

Conflict of interest: None declared.

Open access: This article is available in open access under Creative Common Attribution-Non-Commercial-No Derivatives 4.0 International (CC BY-NC-ND 4.0) license, allowing to download articles and share them with others as long as they credit the authors and the publisher, but without permission to change them in any way or use them commercially. For commercial use, please contact the journal office at kardiologiapolska@ptkardio.pl.

How to cite: Protasiewicz M, Kosowski M, Onisk G, et al. Bioprosthetic aortic scallop intentional laceration to prevent iatrogenic coronary artery obstruction (BASILICA): the first experience in Poland. Kardiol Pol. 2021; 79(10): 1149-1150, doi: 10.33963/KP.a2021.0069.

\section{REFERENCES}

1. Kochman J, KołtowskiŁ, HuczekZ, et al. Complete percutaneous approach versus surgical access in transfemoral transcatheter aortic valve implantation: results from a multicentre registry. Kardiol Pol. 2018; 76(1): 202-208, doi: 10.5603/KP.a2017.0205, indexed in Pubmed: 29131296.

2. Khan JM, Dvir D, Greenbaum AB, et al. Transcatheter laceration of aortic leaflets to prevent coronary obstruction during transcatheter aortic valve replacement: concept to first-in-human. JACC Cardiovasc Interv. 2018; 11(7): 677-689, doi: 10.1016/j.jcin.2018.01.247, indexed in Pubmed: 29622147.

3. Khan JM, Greenbaum AB, Babaliaros VC, et al. The BASILICA trial: prospective multicenter investigation of intentional leaflet laceration to prevent TAVR coronary obstruction. JACC Cardiovasc Interv. 2019; 12(13): 1240 1252, doi: 10.1016/j.jcin.2019.03.035, indexed in Pubmed: 31202947.

4. Protasiewicz M, Szymkiewicz P, Kuliczkowski W, et al. Modern antiplatelet therapy — opportunities and risks. Adv Clin Exp Med. 2013;22(6):875-885, indexed in Pubmed: 24431318.

5. Piroli F, Franchin L, Bruno F, et al. New advances in the prevention of transcatheter aortic valve implantation failure: current and future perspectives. Kardiol Pol. 2020; 78(9): 842-849, doi: 10.33963/KP.15522, indexed in Pubmed: 32692029. 